Spher i cal square functi ons of Marci nki ewi cz type with Ri esz potential s

\begin{tabular}{|l|l|}
\hline 著者 & Sat o Shui chi \\
\hline $\begin{array}{l}\text { j our nal or } \\
\text { publ i cat i on t i t l e }\end{array}$ & Ar chi v der Nat hemat i k \\
\hline vol une & 108 \\
\hline nunber & 4 \\
\hline page r ange & $415-426$ \\
\hline year & 2017- 04-01 \\
\hline URL & ht t p: //hdl . handl e. net /2297/47026 \\
\hline
\end{tabular}




\title{
SPHERICAL SQUARE FUNCTIONS OF MARCINKIEWICZ TYPE WITH RIESZ POTENTIALS
}

\author{
SHUICHI SATO
}

\begin{abstract}
We prove a pointwise equivalence between a spherical square function composed with the Riesz potential and a Littlewood-Paley function arising from the Bochner-Riesz operators. Also, its application to the theory of Sobolev spaces will be given.
\end{abstract}

\section{INTRODUCTION}

Let

$$
\nu(f)(x)=\left(\int_{0}^{\infty}|f(x+t)+f(x-t)-2 f(x)|^{2} \frac{d t}{t^{3}}\right)^{1 / 2} .
$$

Then $\mu(f)=\nu(\mathcal{J}(f))$ is the function of Marcinkiewicz, where $\mathcal{T}(f)(x)=\int_{0}^{x} f(y) d y$. If $f \in L^{p}(\mathbb{R}), 1<p<\infty$, we have

$$
\|\mu(f)\|_{p} \simeq\|f\|_{p}
$$

which means that there exist positive constants $c_{1}, c_{2}$ independent of $f$ such that

$$
c_{1}\|f\|_{p} \leq\|\mu(f)\|_{p} \leq c_{2}\|f\|_{p} .
$$

In other words, we have $\|\nu(f)\|_{p} \simeq\left\|f^{\prime}\right\|_{p}$ if $f$ is in the Sobolev space $W^{1, p}(\mathbb{R})$, $1<p<\infty$.

The Marcinkiewicz function was introduced by J. Marcinkiewicz [5] in 1938 in the setting of periodic functions on the torus $\mathbb{T}$. It can be used to investigate properties of functions such as differentiability and finiteness of norms in function spaces. Zygmund [10] proved a periodic analogue of (1.1), which was conjectured in [5]. The non-periodic version (1.1) was proved by Waterman [8].

The Marcinkiewicz function is a kind of Littlewood-Paley functions; $\mu(f)$ can be realized as

$$
\mu(f)(x)=\left(\int_{0}^{\infty}\left|\psi_{t} * f(x)\right|^{2} \frac{d t}{t}\right)^{1 / 2},
$$

where $\psi_{t}(x)=t^{-1} \psi\left(t^{-1} x\right)$ with $\psi(x)=\chi_{[0,1]}(x)-\chi_{[-1,0]}(x)$; here $\chi_{E}$ denotes the characteristic function of a set $E$. We observe that

$$
f(x+t)+f(x-t)-2 f(x)=\int_{S^{0}}(f(x-t \theta)-f(x)) d \sigma(\theta)
$$

where $d \sigma$ is a uniform measure on $S^{0}=\{-1,1\}$ such that $\sigma(\{-1\})=1, \sigma(\{1\})=1$.

2010 Mathematics Subject Classification. Primary 42B25.

Key Words and Phrases. Littlewood-Paley function, Marcinkiewicz function, Sobolev space.

The author is partly supported by Grant-in-Aid for Scientific Research (C) No. 25400130, Japan Society for the Promotion of Science. 
In this note we assume that $n \geq 2$ and consider the square function

$$
D^{\alpha}(f)(x)=\left(\int_{0}^{\infty}\left|t^{-\alpha} \int_{S^{n-1}}(f(x-t \theta)-f(x)) d \sigma(\theta)\right|^{2} \frac{d t}{t}\right)^{1 / 2},
$$

for appropriate functions $f$, where $d \sigma$ is the Lebesgue surface measure on $S^{n-1}$. Then $D^{\alpha}(f)$ with $\alpha=1$ can be regarded as a generalization to higher dimensions of $\nu(f)$. We shall see that $D^{\alpha}(f)$ also can be used to characterize Sobolev norms. This will be accomplished through a relation between $D^{\alpha}(f)$ and another square function arising from the Bochner-Riesz operators. Let

$$
S_{R}^{\beta}(f)(x)=\int_{|\xi|<R} \widehat{f}(\xi)\left(1-R^{-2}|\xi|^{2}\right)^{\beta} e^{2 \pi i\langle x, \xi\rangle} d \xi=H_{R^{-1}}^{\beta} * f(x)
$$

be the Bochner-Riesz mean of order $\beta$ on $\mathbb{R}^{n}$, where

$$
H_{R^{-1}}^{\beta}(x)=R^{n} H^{\beta}(R x), \quad H^{\beta}(x)=\pi^{-\beta} \Gamma(\beta+1)|x|^{-(n / 2+\beta)} J_{n / 2+\beta}(2 \pi|x|)
$$

with $J_{\nu}$ denoting the Bessel function of the first kind of order $\nu$ and $\hat{f}$ is the Fourier transform defined as

$$
\hat{f}(\xi)=\int_{\mathbb{R}^{n}} f(x) e^{-2 \pi i\langle x, \xi\rangle} d x, \quad\langle x, \xi\rangle=\sum_{k=1}^{n} x_{k} \xi_{k} .
$$

We recall a Littlewood-Paley operator $\sigma_{\beta}, \operatorname{Re}(\beta)>0$, defined from the BochnerRiesz means as

$$
\begin{aligned}
\sigma_{\beta}(f)(x) & =\left(\int_{0}^{\infty}\left|R \partial_{R} S_{R}^{\beta}(f)(x)\right|^{2} \frac{d R}{R}\right)^{1 / 2} \\
& =\left(\int_{0}^{\infty}\left|-2 \beta\left(S_{R}^{\beta}(f)(x)-S_{R}^{\beta-1}(f)(x)\right)\right|^{2} \frac{d R}{R}\right)^{1 / 2},
\end{aligned}
$$

where $\operatorname{Re}(\beta)$ denotes the real part of the complex number $\beta$ and $\partial_{R}=\partial / \partial_{R}$. Also, let $I_{\alpha}$ be the Riesz potential operator defined as

$$
\widehat{I_{\alpha}(f)}(\xi)=|\xi|^{-\alpha} \hat{f}(\xi) .
$$

Let $\mathcal{S}\left(\mathbb{R}^{n}\right)$ be the Schwartz class of rapidly decreasing smooth functions on $\mathbb{R}^{n}$. Let $\mathcal{S}_{0}\left(\mathbb{R}^{n}\right)$ be the subspace of $\mathcal{S}\left(\mathbb{R}^{n}\right)$ consisting of functions $f$ with $\hat{f}$ vanishing in a neighborhood of the origin. We shall prove the following.

Theorem 1.1. Let $0<\alpha<2$. Then if $\beta=\alpha+\frac{n}{2}$, we have

$$
\sigma_{\beta}(f)(x) \approx D^{\alpha}\left(I_{\alpha} f\right)(x)
$$

for $f \in \mathcal{S}_{0}\left(\mathbb{R}^{n}\right)$, where $D^{\alpha}, I_{\alpha}$ and $\sigma_{\beta}$ are as in (1.2), (1.4) and (1.3), respectively.

Here $\sigma_{\beta}(f)(x) \approx D^{\alpha}\left(I_{\alpha} f\right)(x)$ means that there exist positive constants $A, B$ independent of $f$ and $x$ such that

$$
A \sigma_{\beta}(f)(x) \leq D^{\alpha}\left(I_{\alpha} f\right)(x) \leq B \sigma_{\beta}(f)(x) .
$$

A version of Theorem 1.1 was shown in [4] for the range $0<\alpha<1$. In this note we shall extend this range of $\alpha$ to $0<\alpha<2$. The difference from [4] that enables us to improve the range of $\alpha$ mainly comes from the estimate in part (1) of Lemma 2.5 .

In Section 2, we shall give an almost self-contained proof of Theorem 1.1 except that Lemmas 2.2, 2.3 from [9] and the formula (2.9) are taken for granted. In 
Section 3, applications of Theoerm 1.1 to the theory of Sobolev spaces will be given.

\section{Proof of Theorem 1.1}

For a fixed function $f \in \mathcal{S}_{0}\left(\mathbb{R}^{n}\right)$ and a fixed point $x \in \mathbb{R}^{n}$, let

$$
\begin{gathered}
\varphi(t)=\varphi(t ; x, f)=\int_{S^{n-1}} f\left(x-t y^{\prime}\right) d \sigma\left(y^{\prime}\right), \\
\theta(t)=\theta(t ; x, f)=t \frac{\partial}{\partial t} \varphi(t ; x, f)=-\int_{S^{n-1}}\left\langle t y^{\prime}, \nabla f\left(x-t y^{\prime}\right)\right\rangle d \sigma\left(y^{\prime}\right),
\end{gathered}
$$

where $\nabla f(x)=\left(\partial_{1} f(x), \ldots, \partial_{n} f(x)\right), \partial_{j}=\partial / \partial x_{j}$.

Let $\operatorname{Re} \alpha>-n$. Define

$$
\widehat{I^{\alpha}(f)}(\xi)=|\xi|^{\alpha} \hat{f}(\xi)
$$

and for $\operatorname{Re} \beta>-1$ consider

$$
S_{R}^{\beta}\left(I^{\alpha} f\right)(x)=\int_{|\xi|<R} \widehat{f}(\xi)|\xi|^{\alpha}\left(1-R^{-2}|\xi|^{2}\right)^{\beta} e^{2 \pi i\langle x, \xi\rangle} d \xi=R^{\alpha} L_{R^{-1}}^{\alpha, \beta} * f(x),
$$

where

$$
\begin{aligned}
L^{\alpha, \beta}(x) & =\int_{|\xi|<1}|\xi|^{\alpha}\left(1-|\xi|^{2}\right)^{\beta} e^{2 \pi i\langle x, \xi\rangle} d \xi \\
& =\int_{0}^{1} r^{n+\alpha}\left(1-r^{2}\right)^{\beta} 2 \pi(r|x|)^{-(n-2) / 2} J_{(n-2) / 2}(2 \pi r|x|) \frac{d r}{r} \\
& =(2 \pi)^{n / 2} \int_{0}^{1} r^{n+\alpha}\left(1-r^{2}\right)^{\beta} V_{(n-2) / 2}(2 \pi r|x|) \frac{d r}{r}
\end{aligned}
$$

with

$$
V_{\nu}(r)=r^{-\nu} J_{\nu}(r) .
$$

We write the formula in (2.4) by using $\varphi$ in (2.1).

Lemma 2.1. Suppose that $\operatorname{Re} \alpha>-n, \operatorname{Re} \beta>-1$. Let

$$
l_{\alpha, \beta}(s)=(2 \pi)^{n / 2} \int_{0}^{1} r^{n+\alpha}\left(1-r^{2}\right)^{\beta} V_{(n-2) / 2}(2 \pi r s) \frac{d r}{r} .
$$

Then we have

$$
S_{R}^{\beta}\left(I^{\alpha} f\right)(x)=R^{n+\alpha} \int_{0}^{\infty} l_{\alpha, \beta}(R s) \varphi(s ; x, f) s^{n} \frac{d s}{s} .
$$

Proof. By (2.4) we have

$$
S_{R}^{\beta}\left(I^{\alpha} f\right)(x)=R^{\alpha} L_{R^{-1}}^{\alpha, \beta} * f(x)=R^{\alpha} \int_{\mathbb{R}^{n}} f(x-y) L_{R^{-1}}^{\alpha, \beta}(y) d y .
$$

Using polar coordinates and recalling the definition of $L^{\alpha, \beta}$ in the last integral, by (2.1) we reach the conclusion.

We use the following formulas. 
Lemma 2.2. If $\operatorname{Re}(\mu)>-1, \operatorname{Re}(\nu)>-1, t>0$,

$$
J_{\mu+\nu+1}(t)=\frac{t^{\nu+1}}{2^{\nu} \Gamma(\nu+1)} \int_{0}^{1} J_{\mu}(t s) s^{\mu+1}\left(1-s^{2}\right)^{\nu} d s .
$$

This can be rewritten as

$$
V_{\mu+\nu+1}(t)=\frac{1}{2^{\nu} \Gamma(\nu+1)} \int_{0}^{1} V_{\mu}(t s) s^{2 \mu+1}\left(1-s^{2}\right)^{\nu} d s .
$$

Lemma 2.3. If $0<\operatorname{Re}(\mu)<\operatorname{Re}(\nu)+1 / 2$,

$$
\int_{0}^{\infty} t^{\mu-1} V_{\nu}(t) d t=\frac{\Gamma(\mu / 2)}{2^{\nu-\mu+1} \Gamma(\nu-\mu / 2+1)} .
$$

See [9, p.373] and [9, p.391] for Lemma 2.2 and Lemma 2.3, respectively.

We need the expression of $\sigma_{\beta}\left(I^{\alpha} f\right)$ in (2.7) below. To obtain it we show the following.

Lemma 2.4. Let $\operatorname{Re} \alpha>-n, \operatorname{Re} \beta>0$. Then

$$
\begin{aligned}
& R \partial_{R} S_{R}^{\beta}\left(I^{\alpha} f\right)(x) \\
& \quad=-2 \beta(2 \pi)^{n / 2} R^{\alpha} \int_{0}^{\infty} \theta(s / R) s^{n-1} d s \int_{0}^{1} r^{n+\alpha+2}\left(1-r^{2}\right)^{\beta-1} V_{n / 2}(2 \pi r s) \frac{d r}{r} .
\end{aligned}
$$

Proof. We first compute $R \partial_{R}\left(R^{n+\alpha} l_{\beta}(R s)\right)$ as follows.

$$
\begin{aligned}
R \partial_{R}\left(R^{n+\alpha} l_{\alpha, \beta}(R s)\right) & =R \partial_{R}\left[(2 \pi)^{n / 2} R^{n+\alpha} \int_{0}^{1} r^{n+\alpha}\left(1-r^{2}\right)^{\beta} V_{(n-2) / 2}(2 \pi R r s) \frac{d r}{r}\right] \\
& =R \partial_{R}\left[(2 \pi)^{n / 2} \int_{0}^{R} r^{n+\alpha}\left(1-\frac{r^{2}}{R^{2}}\right)^{\beta} V_{(n-2) / 2}(2 \pi r s) \frac{d r}{r}\right] \\
& =R(2 \pi)^{n / 2} \int_{0}^{R} r^{n+\alpha} \beta\left(1-\frac{r^{2}}{R^{2}}\right)^{\beta-1}\left(2 r^{2} R^{-3}\right) V_{(n-2) / 2}(2 \pi r s) \frac{d r}{r} \\
& =2 \beta(2 \pi)^{n / 2} R^{n+\alpha} \int_{0}^{1} r^{n+\alpha}\left(1-r^{2}\right)^{\beta-1} r^{2} V_{(n-2) / 2}(2 \pi R r s) \frac{d r}{r} .
\end{aligned}
$$

Therefore, from Lemma 2.1 it follows that

$$
=2 \beta(2 \pi)^{n / 2} R^{n+\alpha} \int_{0}^{\infty} \varphi(s ; x, f) s^{n-1} d s \int_{0}^{1} r^{n+\alpha}\left(1-r^{2}\right)^{\beta-1} r^{2} V_{(n-2) / 2}(2 \pi R r s) \frac{d r}{r} .
$$

By Lemma 2.2 we have

$$
\begin{aligned}
\int_{0}^{u} V_{(n-2) / 2}(2 \pi R r s) s^{n-1} d s & =u^{n} \int_{0}^{1} V_{(n-2) / 2}(2 \pi R r s u) s^{n-1} d s \\
& =u^{n} \Gamma(1) V_{n / 2}(2 \pi r R u)=u^{n} V_{n / 2}(2 \pi r R u) .
\end{aligned}
$$


Thus applying integration by parts in (2.5), we see that

$$
\begin{aligned}
& R \partial_{R} S_{R}^{\beta}\left(I^{\alpha} f\right)(x) \\
& =-2 \beta(2 \pi)^{n / 2} R^{n+\alpha} \int_{0}^{\infty} \varphi^{\prime}(s) d s \int_{0}^{1} r^{n+\alpha}\left(1-r^{2}\right)^{\beta-1} r^{2} s^{n} V_{n / 2}(2 \pi R r s) \frac{d r}{r} \\
& =-2 \beta(2 \pi)^{n / 2} R^{n+\alpha} \int_{0}^{\infty} \theta(s) s^{n-1} d s \int_{0}^{1} r^{n+\alpha}\left(1-r^{2}\right)^{\beta-1} r^{2} V_{n / 2}(2 \pi R r s) \frac{d r}{r} \\
& =-2 \beta(2 \pi)^{n / 2} R^{\alpha} \int_{0}^{\infty} \theta(s / R) s^{n-1} d s \int_{0}^{1} r^{n+\alpha+2}\left(1-r^{2}\right)^{\beta-1} V_{n / 2}(2 \pi r s) \frac{d r}{r}
\end{aligned}
$$

where $\varphi(s)=\varphi(s ; x, f)$ and $\theta(s)=\theta(s ; x, f)$ are as in (2.1) and (2.2), respectively. This completes the proof.

For appropriate complex numbers $\alpha, \beta$, let

$$
\Phi_{\alpha, \beta}(s)=s^{\alpha+n} \int_{0}^{1} r^{n+\alpha+1}\left(1-r^{2}\right)^{\beta-1} V_{n / 2}(2 \pi r s) d r
$$

and

$$
\theta_{\alpha}(t)=t^{-\alpha} \theta(t)=t^{-\alpha} \theta(t ; x, f) .
$$

Then Lemma 2.4 implies that

$$
R \partial_{R} S_{R}^{\beta}\left(I^{\alpha} f\right)(x)=-2 \beta(2 \pi)^{n / 2} \int_{0}^{\infty} \Phi_{\alpha, \beta}(s) \theta_{\alpha}\left(s R^{-1}\right) \frac{d s}{s}
$$

for $\operatorname{Re} \alpha>-n, \operatorname{Re} \beta>0$. Define

$$
\begin{gathered}
K_{\alpha, \beta}(u)=-2 \beta(2 \pi)^{n / 2} \Phi_{\alpha, \beta}\left(e^{u}\right), \\
\Theta_{\alpha}(u)=\Theta_{\alpha}(u, x, f)=\theta_{\alpha}\left(e^{-u}\right), \quad \Theta(u)=\theta\left(e^{-u}\right) .
\end{gathered}
$$

Then by change of variables $s=e^{v}, R=e^{u}$ we have

$$
R \partial_{R} S_{R}^{\beta}\left(I^{\alpha} f\right)(x)=\int_{-\infty}^{\infty} K_{\alpha, \beta}(v) \Theta_{\alpha}(u-v) d v,
$$

and hence by (1.3),

$$
\sigma_{\beta}\left(I^{\alpha} f\right)(x)^{2}=\int_{-\infty}^{\infty}\left|K_{\alpha, \beta} * \Theta_{\alpha}(u)\right|^{2} d u .
$$

In proving Theorem 1.1 we need Proposition 2.10 below. To show it we first state some properties of $\Theta_{\alpha}$ and $K_{\alpha, \beta}$ (Lemmas 2.5 and 2.6).

Lemma 2.5. We have the following estimates for $\Theta_{\alpha}, \alpha \in \mathbb{C}$.

(1) $\left|\Theta_{\alpha}(u)\right| \leq C e^{u \operatorname{Re}(\alpha)} e^{-2 u}$ for $u \geq 0$;

(2) if $u<0,\left|\Theta_{\alpha}(u)\right| \leq C_{m} e^{u \operatorname{Re}(\alpha)} e^{m u}$ for any $m>0$.

Further, we have similar estimates for the derivatives $(d / d u)^{k} \Theta_{\alpha}, k=1,2, \ldots$ In particular, $\Theta_{\alpha} \in \mathcal{S}(\mathbb{R})$ if $\operatorname{Re}(\alpha)<2$.

Proof. Recall that $\theta(t)=-\int_{S^{n-1}}\left\langle t y^{\prime}, \nabla f\left(x-t y^{\prime}\right)\right\rangle d \sigma\left(y^{\prime}\right)$ and $\Theta_{\alpha}(u)=e^{u \alpha} \theta\left(e^{-u}\right)$. Thus, part (2) follows easily since $|\nabla f(x)| \leq C_{m}(1+|x|)^{-m-1}$ and $\left|x-e^{-u} y^{\prime}\right| \geq$ $e^{-u}-|x| \geq e^{-u} / 2$ if $e^{-u} \geq 2|x|$.

To prove part (1), we note that

$$
\theta(t)=-\int_{S^{n-1}}\left\langle t y^{\prime}, \nabla f\left(x-t y^{\prime}\right)-\nabla f(x)\right\rangle d \sigma\left(y^{\prime}\right),
$$


since $\int_{S^{n-1}}\left\langle t y^{\prime}, w\right\rangle d \sigma\left(y^{\prime}\right)=0$ for any $w \in \mathbb{R}^{n}$. So $\theta(t)=O\left(t^{2}\right)$ as $t \rightarrow 0$, which proves part (1).

By a direct computation, we can prove the result for the derivatives $(d / d u)^{k} \Theta_{\alpha}$ similarly.

Lemma 2.6. The following results hold for $K_{\alpha, \beta}, \alpha, \beta \in \mathbb{C}$.

(1) If $\operatorname{Re}(\alpha)>-n-2$ and $\operatorname{Re}(\beta)>0$,

$$
\left|K_{\alpha, \beta}(u)\right| \leq C_{\alpha, \beta} e^{(n+\operatorname{Re}(\alpha)) u}, \quad u \in \mathbb{R} .
$$

(2) If $\operatorname{Re}(\alpha)>-n / 2-2$ and $\operatorname{Re}(\beta)>0$,

$$
\left|K_{\alpha, \beta}(u)\right| \leq C_{\alpha, \beta} e^{(n / 2+\operatorname{Re}(\alpha)) u}, \quad u \in \mathbb{R} .
$$

(3) If $-n / 2>\operatorname{Re}(\alpha)>-n / 2-1$ and $\operatorname{Re}(\beta)>0$, then

$$
\left|K_{\alpha, \beta}(u)\right| \leq C_{\alpha, \beta} e^{-\delta|u|}, \quad u \in \mathbb{R},
$$

where $\delta=\min (n+\operatorname{Re}(\alpha),-\operatorname{Re}(\alpha)-n / 2)>0$.

Proof. Since $V_{n / 2}$ is bounded, by (2.6) we have part (1), where we assume that $\operatorname{Re}(\alpha)>-n-2$ and $\operatorname{Re}(\beta)>0$ for the integrabilities on $[0,1]$ of $r^{n+\alpha+1}$ and $\left(1-r^{2}\right)^{\beta-1}$, respectively. By $(2.6)$, we also have

$$
\Phi_{\alpha, \beta}(s)=(2 \pi)^{-n / 2} s^{\alpha+n / 2} \int_{0}^{1} r^{n / 2+\alpha+1}\left(1-r^{2}\right)^{\beta-1} J_{n / 2}(2 \pi r s) d r .
$$

This implies part (2). Part (3) follows from the estimates of (1) and (2).

Let

$$
G(\alpha, \beta)=\int_{-\infty}^{\infty} K_{\alpha, \beta} * \Theta_{\alpha}(u) g(u) d u,
$$

where $g \in C_{0}^{\infty}(\mathbb{R})$. By Lemmas 2.5 and 2.6, the convolution $K_{\alpha, \beta} * \Theta_{\alpha}$ can be defined and $G(\alpha, \beta)$ is analytic in $\alpha$ and $\beta$ if $\operatorname{Re}(\alpha)>-n-2, \operatorname{Re}(\beta)>0$. Also, if $-n / 2>\operatorname{Re}(\alpha)>-n / 2-1$ and $\operatorname{Re}(\beta)>0$, then $K_{\alpha, \beta}, \Theta_{\alpha} \in L^{1}(\mathbb{R}) ;$ in this case we have

$$
G(\alpha, \beta)=\int_{-\infty}^{\infty} \widehat{K}_{\alpha, \beta}(\xi) \widehat{\Theta}_{\alpha}(\xi) \hat{g}(-\xi) d \xi
$$

An explicit form of the Fourier transform of $K_{\alpha, \beta}$ needed is stated in the next result.

Lemma 2.7. If $-n / 2-1<\operatorname{Re}(\alpha)<-n / 2$ and $\operatorname{Re}(\beta)>0$, then $\widehat{K}_{\alpha, \beta}(\xi)=\Psi_{\alpha, \beta}(\xi)$, where

$$
\Psi_{\alpha, \beta}(\xi)=-2^{-1} \beta \pi^{-n / 2-\alpha+2 \pi i \xi} \frac{\Gamma((n+\alpha-2 \pi i \xi) / 2) \Gamma(\pi i \xi+1) \Gamma(\beta)}{\Gamma(-\alpha / 2+\pi i \xi+1) \Gamma(\pi i \xi+1+\beta)} .
$$


Proof. Let $-\operatorname{Re}(\alpha)-n<\operatorname{Re}(\zeta)<-\operatorname{Re}(\alpha)-n / 2$ and $-n / 2-1<\operatorname{Re}(\alpha)<-n / 2$, $\operatorname{Re}(\beta)>0$. Then by Fubini's theorem we have

$$
\begin{aligned}
& \widehat{K}_{\alpha, \beta}\left(\frac{\zeta}{-2 \pi i}\right)=\int_{-\infty}^{\infty} e^{\zeta u} K_{\alpha, \beta}(u) d u \\
& =-2 \beta(2 \pi)^{n / 2} \int_{0}^{\infty} t^{n+\alpha+\zeta-1}\left(\int_{0}^{1} r^{n+\alpha+1}\left(1-r^{2}\right)^{\beta-1} V_{n / 2}(2 \pi r t) d r\right) d t \\
& =-2 \beta(2 \pi)^{n / 2} \int_{0}^{1} r^{n+\alpha+1}\left(1-r^{2}\right)^{\beta-1}\left(\int_{0}^{\infty} t^{n+\alpha+\zeta-1} V_{n / 2}(2 \pi r t) d t\right) d r .
\end{aligned}
$$

Using Lemma 2.3, we see that

$$
\begin{aligned}
& \int_{0}^{\infty} t^{n+\alpha+\zeta-1} V_{n / 2}(2 \pi r t) d t=(2 \pi r)^{-n-\alpha-\zeta} \int_{0}^{\infty} t^{n+\alpha+\zeta-1} V_{n / 2}(t) d t \\
& =(2 \pi)^{-n-\alpha-\zeta} 2^{n / 2+\alpha+\zeta-1} \frac{\Gamma((n+\alpha+\zeta) / 2)}{\Gamma(-\alpha / 2-\zeta / 2+1)} r^{-n-\alpha-\zeta}
\end{aligned}
$$

Thus

$$
\begin{aligned}
& \widehat{K}_{\alpha, \beta}\left(\frac{\zeta}{-2 \pi i}\right) \\
& =-2 \beta(2 \pi)^{-n / 2-\alpha-\zeta} 2^{n / 2+\alpha+\zeta-1} \frac{\Gamma((n+\alpha+\zeta) / 2)}{\Gamma(-\alpha / 2-\zeta / 2+1)} \int_{0}^{1} r^{1-\zeta}\left(1-r^{2}\right)^{\beta-1} d r \\
& =-\beta \pi^{-n / 2-\alpha-\zeta} \frac{\Gamma((n+\alpha+\zeta) / 2)}{\Gamma(-\alpha / 2-\zeta / 2+1)} 2^{-1} \int_{0}^{1} t^{-\zeta / 2}(1-t)^{\beta-1} d t \\
& =-2^{-1} \beta \pi^{-n / 2-\alpha-\zeta} \frac{\Gamma((n+\alpha+\zeta) / 2)}{\Gamma(-\alpha / 2-\zeta / 2+1)} \frac{\Gamma(-\zeta / 2+1) \Gamma(\beta)}{\Gamma(-\zeta / 2+1+\beta)} .
\end{aligned}
$$

Putting $\zeta=-2 \pi i \xi, \xi \in \mathbb{R}$, we reach the conclusion.

In proving Proposition 2.10 we also need the following.

Lemma 2.8. Let $\Psi_{\alpha, \beta}$ be as in Lemma 2.7 and

$$
H(\alpha, \beta)=\int_{-\infty}^{\infty} \Psi_{\alpha, \beta}(\xi) \widehat{\Theta}_{\alpha}(\xi) \hat{g}(-\xi) d \xi
$$

Then $H(\alpha, \beta)$ is analytic in $\alpha$ and $\beta$ if $-n<\operatorname{Re}(\alpha)<2, \operatorname{Re}(\beta)>0$.

To prove this we apply the following.

Lemma 2.9 (asymptotic formula for the gamma function). Let $a, \xi \in \mathbb{R}, a>0$. Then we have

$$
\lim _{|\xi| \rightarrow \infty} \frac{|\Gamma(a+i \xi)|}{\sqrt{2 \pi} e^{-\pi|\xi| / 2}|\xi|^{a-1 / 2}}=1 .
$$

This is well-known. In Section 4, we shall give a proof for completeness based on the formula

$$
\lim _{\operatorname{Re}(z) \geq c>0,|z| \rightarrow \infty} \frac{\Gamma(z)}{\sqrt{2 \pi} e^{-z} z^{z-1 / 2}}=1 .
$$

Proof of Lemma 2.8. We can see that $\Psi_{\alpha, \beta}(\xi)$ is analytic in $\alpha, \beta$ for $-n<\operatorname{Re}(\alpha)<$ $2, \operatorname{Re}(\beta)>0$, if $\xi$ is fixed. By Lemma $2.9,\left|\Psi_{\alpha, \beta}(\xi)\right|$ behaves like $|\xi|^{\operatorname{Re}(\alpha)-\operatorname{Re}(\beta)+n / 2-1}$ 
if $|\xi|$ is sufficiently large. Also we note that $\Psi_{\alpha, \beta}(\xi)$ is continuous and does not vanish in $\alpha, \beta, \xi$ with $-n<\operatorname{Re}(\alpha)<2, \operatorname{Re}(\beta)>0, \xi \in \mathbb{R}^{n}$. Thus we have

$$
A(1+|\xi|)^{\operatorname{Re}(\alpha)-\operatorname{Re}(\beta)+n / 2-1} \leq\left|\Psi_{\alpha, \beta}(\xi)\right| \leq B(1+|\xi|)^{\operatorname{Re}(\alpha)-\operatorname{Re}(\beta)+n / 2-1}
$$

with some positive numbers $A$ and $B$ independent of $\xi$.

Using (2.10), since $\hat{g} \in \mathcal{S}(\mathbb{R})$ and $\widehat{\Theta}_{\alpha}$ is bounded and analytic for $\operatorname{Re}(\alpha)<2$ by part (1) of Lemma 2.5, we can see $H(\alpha, \beta)$ is analytic for $-n<\operatorname{Re}(\alpha)<2$, $\operatorname{Re}(\beta)>0$.

Now we are able to prove the following.

Proposition 2.10. Let $-n<\operatorname{Re}(\alpha)<2, \operatorname{Re}(\beta)>0$. Then

$$
\sigma_{\beta}\left(I^{\alpha} f\right)(x)^{2}=\int_{-\infty}^{\infty}\left|K_{\alpha, \beta} * \Theta_{\alpha}(u)\right|^{2} d u=\int_{-\infty}^{\infty}\left|\Psi_{\alpha, \beta}(\xi)\right|^{2}\left|\widehat{\Theta}_{\alpha}(\xi)\right|^{2} d \xi .
$$

Proof. We recall that $G(\alpha, \beta)$ in (2.8) is analytic in $\alpha$ and $\beta$ for $\operatorname{Re}(\alpha)>-n-2$, $\operatorname{Re}(\beta)>0$ and that $H(\alpha, \beta)$ is analytic for $-n<\operatorname{Re}(\alpha)<2, \operatorname{Re}(\beta)>0$ (Lemma 2.8). Further, by Lemma 2.7 we have $G(\alpha, \beta)=H(\alpha, \beta)$ if $-n / 2-1<\operatorname{Re}(\alpha)<$ $-n / 2$ and $\operatorname{Re}(\beta)>0$. Thus by analytic continuation we see that $G(\alpha, \beta)=H(\alpha, \beta)$ if $-n<\operatorname{Re}(\alpha)<2, \operatorname{Re}(\beta)>0$. So, we have $|G(\alpha, \beta)|=|H(\alpha, \beta)|$ if $-n<\operatorname{Re}(\alpha)<$ $2, \operatorname{Re}(\beta)>0$. Thus, taking the supremum over $g$ in the unit ball of $L^{2}(\mathbb{R})$ and recalling (2.7), we get the conclusion.

On the other hand, for $D^{\alpha}(f)$ in (1.2) we have the following result.

Proposition 2.11. Let $\Theta_{\alpha}$ be as in Lemma 2.5. Suppose that $0<\operatorname{Re}(\alpha)<2$. Then

$$
D^{\alpha}(f)(x)^{2}=\int_{-\infty}^{\infty}|\alpha-2 \pi i \xi|^{-2}\left|\widehat{\Theta}_{\alpha}(\xi)\right|^{2} d \xi .
$$

Proof. We note that

$$
\int_{S^{n-1}}(f(x-t \theta)-f(x)) d \sigma(\theta)=\varphi(t ; x, f)-\varphi(0 ; x, f)=\int_{0}^{1} \theta(t r ; x, f) \frac{d r}{r}
$$

and

$$
t^{-\alpha}(\varphi(t ; x, f)-\varphi(0 ; x, f))=\int_{0}^{1} r^{\alpha} \theta_{\alpha}(t r ; x, f) \frac{d r}{r} .
$$

Thus

$$
D^{\alpha}(f)(x)=\left(\int_{0}^{\infty}\left|\int_{0}^{1} r^{\alpha} \theta_{\alpha}(t r ; x, f) \frac{d r}{r}\right|^{2} \frac{d t}{t}\right)^{1 / 2} .
$$

By the change of variables $r=e^{v}, t=e^{-u}$, we have

$$
D^{\alpha}(f)(x)^{2}=\int_{-\infty}^{\infty}\left|\int_{-\infty}^{0} e^{\alpha v} \Theta_{\alpha}(u-v) d v\right|^{2} d u=\int_{-\infty}^{\infty}\left|\hat{\psi}_{\alpha}(\xi)\right|^{2}\left|\widehat{\Theta}_{\alpha}(\xi)\right|^{2} d \xi
$$

where $\psi_{\alpha}(u)=e^{\alpha u} \chi_{\{u \leq 0\}}(u)$ and hence

$$
\hat{\psi}_{\alpha}(\xi)=\int_{-\infty}^{0} e^{\alpha u} e^{-2 \pi i u \xi} d u=\frac{1}{\alpha-2 \pi i \xi} .
$$

Here we note that $\psi_{\alpha}$ and $\Theta_{\alpha}$ are in $L^{1}(\mathbb{R})$ and in $L^{2}(\mathbb{R})$ if $0<\operatorname{Re}(\alpha)<2$ (see Lemma 2.5 for $\Theta_{\alpha}$ ). This completes the proof. 
Proof of Theorem 1.1. Let $0<\alpha<2$. Then we note that

$$
(\alpha / 2)(1+|\xi|) \leq|\alpha+2 \pi i \xi| \leq 2 \pi(1+|\xi|) .
$$

By this and (2.10) it follows that $|\alpha-2 \pi i \xi|^{-1}$ and $\left|\Psi_{\alpha, \beta}(\xi)\right|$ are pointwise equivalent as functions of $\xi$ if $\beta=\alpha+n / 2$. Using the pointwise equivalence and the formulas of Propositions 2.10 and 2.11, we have $\sigma_{\beta}\left(I^{\alpha} f\right)(x) \approx D^{\alpha}(f)(x)$ for $f \in \mathcal{S}_{0}\left(\mathbb{R}^{n}\right)$. Substituting $I_{\alpha} f$ for $f$ and recalling (1.4), (2.3), we reach the conclusion.

\section{Applications}

Let $D^{\alpha}, I_{\alpha}$ and $\sigma_{\beta}$ be as in (1.2), (1.4) and (1.3), respectively. Define

$$
S_{\alpha}(f)(x)=D^{\alpha}\left(I_{\alpha} f\right)(x)
$$

for $f \in \mathcal{S}\left(\mathbb{R}^{n}\right)$. Then, some $L^{p}$ estimates for $S_{\alpha}, 0<\alpha<2$, are shown in [7] with weights for $1 / 2 \leq \alpha<2$, which are useful in characterizing the Sobolev spaces of order $\alpha$ (see [1] for relevant results). When $1<\alpha<2$, the result is proved by applying a theorem of [6] for the boundedness of Littlewood-Paley operators. When $0<\alpha<1$ it is shown by using Theorem 1.1 for $\alpha \in(0,1)$, which is due to [4], and applying known properties of $\sigma_{\beta}$. The result for the case $\alpha=1$ is due to [3]. Here, we focus on the case $1 / 2 \leq \alpha<2$. Then, more precisely, we can find the following result in [7].

Theorem A. Suppose that $1 / 2 \leq \alpha<2, w \in A_{p}, 1<p<\infty$. Let $S_{\alpha}$ be as in (3.1). Let $f \in \mathcal{S}\left(\mathbb{R}^{n}\right)$. Then

$$
\left\|S_{\alpha}(f)\right\|_{p, w} \simeq\|f\|_{p, w} .
$$

We recall the weight class $A_{p}$ of Muckenhoupt. A weight $w$ belongs to $A_{p}$, $1<p<\infty$, if

$$
\sup _{B}\left(|B|^{-1} \int_{B} w(x) d x\right)\left(|B|^{-1} \int_{B} w(x)^{-1 /(p-1)} d x\right)^{p-1}<\infty,
$$

where the supremum is taken over all balls $B$ in $\mathbb{R}^{n}$ and $|B|$ denotes the Lebesgue measure of $B$ (see [2] for the $A_{p}$ class). The weighted $L^{p}$ space is defined as $L_{w}^{p}$ with the norm

$$
\|f\|_{L_{w}^{p}}=\|f\|_{p, w}=\left(\int_{\mathbb{R}^{n}}|f(x)|^{p} w(x) d x\right)^{1 / p} .
$$

Theorem A is due to [3] when $\alpha=1$, as mentioned above. We can now give a different proof of this by applying Theorem 1.1 as follows. Since it is known that $\sigma_{n / 2+1}$ is bounded on $L_{w}^{p}$ for all $p \in(1, \infty)$ and $w \in A_{p}$ (see [7]), by Theorem 1.1 with $\alpha=1$ we have

$$
\left\|S_{1}(f)\right\|_{p, w} \leq C\|f\|_{p, w}
$$

for $f \in \mathcal{S}\left(\mathbb{R}^{n}\right)$. The reverse inequality follows from this by duality as in [7].

Similarly, we can give another proof of Theorem A for $\alpha \in(1,2)$, which was proved in [7] from a result of [6], by applying Theorem 1.1 and the boundedness of $\sigma_{n / 2+\alpha}$ on $L_{w}^{p}$ with $w \in A_{p}, 1<p<\infty$. 


\section{Proof of Lemma 2.9}

By (2.9) to prove Lemma 2.9 it suffices to show

$$
\begin{aligned}
& \exp \left(-\frac{a^{3}}{a^{2}+\xi^{2}}\right)\left(1+\frac{a^{2}}{\xi^{2}}\right)^{(a-1 / 2) / 2} \\
& \quad \leq \frac{\left|e^{-z} z^{z-1 / 2}\right|}{e^{-\pi|\xi| / 2}|\xi|^{a-1 / 2}} \leq\left(1+\frac{a^{2}}{\xi^{2}}\right)^{(a-1 / 2) / 2},
\end{aligned}
$$

where $z=a+i \xi, \xi \neq 0$.

To prove (4.1), we first note that

$$
\left|z^{z-1 / 2}\right|=e^{(a-1 / 2) \log |z|} e^{-\xi \arg z}=\left(a^{2}+\xi^{2}\right)^{(a-1 / 2) / 2} e^{-|\xi| \arg (a+i|\xi|)},
$$

where $-\pi / 2<\arg z<\pi / 2$. We write $\arg (a+i|\xi|)=\arctan (|\xi| / a)$. Define a function $F$ on $[0, \infty)$ by $F(x)=\arctan (1 / x), x>0, F(0)=\pi / 2$. Then by the mean value theorem, we have

$$
\pi / 2-\arctan (|\xi| / a)=F(0)-F(a /|\xi|)=\frac{a}{|\xi|} \frac{1}{\eta^{2}+1}
$$

for some $\eta \in(0, a /|\xi|)$. Thus

$$
e^{-a} e^{|\xi|(\pi / 2-\arg (a+i|\xi|))}=e^{-a \eta^{2} /\left(\eta^{2}+1\right)}
$$

and hence

$$
\begin{aligned}
\left|e^{-z} z^{z-1 / 2}\right| & =e^{-a}\left(a^{2}+\xi^{2}\right)^{(a-1 / 2) / 2} e^{-\pi|\xi| / 2} e^{|\xi|(\pi / 2-\arg (a+i|\xi|))} \\
& =e^{-\pi|\xi| / 2}|\xi|^{a-1 / 2}\left(1+\frac{a^{2}}{\xi^{2}}\right)^{(a-1 / 2) / 2} e^{-a \eta^{2} /\left(\eta^{2}+1\right)}
\end{aligned}
$$

Since

$$
e^{-a^{3} /\left(a^{2}+\xi^{2}\right)}<e^{-a \eta^{2} /\left(\eta^{2}+1\right)}<1,
$$

from (4.2) we obtain (4.1). This completes the proof of Lemma 2.9.

\section{REFERENCES}

[1] R. Alabern, J. Mateu and J. Verdera, A new characterization of Sobolev spaces on $\mathbb{R}^{n}$, Math. Ann. 354 (2012), 589-626.

[2] J. Garcia-Cuerva and J.L. Rubio de Francia, Weighted Norm Inequalities and Related Topics, North-Holland, Amsterdam, New York, Oxford, 1985.

[3] P. Hajłasz, Z. Liu, A Marcinkiewicz integral type characterization of the Sobolev space, arXiv:1405.6127 [math.FA].

[4] M. Kaneko and G. Sunouchi, On the Littlewood-Paley and Marcinkiewicz functions in higher dimensions, Tôhoku Math. J. 37 (1985), 343-365.

[5] J. Marcinkiewicz, Sur quelues integrales de type de Dini, Annales de la Société Polonaise 17 (1938), 42-50.

[6] S. Sato, Remarks on square functions in the Littlewood-Paley theory, Bull. Austral. Math. Soc. 58 (1998), 199-211.

[7] S. Sato, Littlewood-Paley operators and Sobolev spaces, Illinois J. Math. 58 (2014), 1025-1039.

[8] D. Waterman, On an integral of Marcinkiewicz, Trans. Amer. Math. Soc. 91 (1959), 129-138.

[9] G.N. Watson, A Treatise on the Theory of Bessel Functions, Cambridge Univ. Press, London, 1966.

[10] A. Zygmund, On certain integrals, Trans. Amer. Math. Soc. 58 (1944), 170-204.

Department of Mathematics, Faculty of Education, Kanazawa University, Kanazawa 920-1192, JAPAN

E-mail address: shuichi@kenroku.kanazawa-u.ac.jp 